\title{
A60 CONTRIBUTION OF MESENCHYMAL STEM CELLS, OR FIBROBLAST-LIKE SYNOVIOCYTES TO THE VICIOUS CIRCLE LEADING TO CHRONIC INFLAMMATION IN RA THROUGH PROMOTION OF TH17 CELLS
}

Assia Eljaafari, Marie-Laure Tartelin, Pierre Miossec Immunogenomics and Inflammation Unit, EA4130, Hospices Civils de Lyon and University Claude Bernard Lyon 1

\subsection{6/ard.2010.149104.4}

Background and objectives TH17 cells have been involved in the initiation and maintenance of inflammatory and autoimmune diseases, such as rheumatoid arthritis (RA).

In this study, we hypothesised that interaction of $\mathrm{T}$ cells with mesenchymal cells, such as synoviocytes (FLS), or mesenchymal stem cells (MSC) could participate in the activation of the TH17 pathway, with the help of the inflammatory environment.

Methods To investigate this possibility, peripheral blood mononuclear cells (MNC) from RA patients, or from healthy blood donors, were co-cultured with FLS issued from either $\mathrm{RA}$ or osteoarthritic (OA) patients, or with MSC issued from healthy bone marrow donors. In addition, synovial $T$ cells were cocultured with autologous RA-FLS. Cocultures were exposed to PHA for 1-2 days. IL17-A, TNF $\alpha$ and or/IFN $\gamma$ were added or not to these co-cultures.Q-RT-PCR,ELISA, and cytoflurometry techniques were used to assess for IL17-A production.

Results We observed that the interaction of healthy MNC with MSC resulted in an extinction of TH1 and TH2 cell activation, but induced a strong production of IL-17A, as soon as 24 $h$ post activation, as assessed by (1) the increase of RORC, and IL17-A at the mRNA levels, (2) IL17A secretion in Elisa assays, and (3) Th17 staining, by flow cytometry. The levels of IL17A secretion further increased at $48 \mathrm{~h}$. Moreover, IL6, IL8 and IL1 $\beta$ mRNA levels increased as well, and were potentiated when IL17-A, TNF $\alpha$, and or/IFN $\gamma$ cytokines were added in these co-cultures. Interestingly, co-cultures of RA-MNC or healthy MNC with FLS from either OA or RA patients, also resulted in a strong induction of IL17-A, but not in a complete extinction of TH1 cytokines. In the presence of autologous FLS, RA-synovial T cells increased their levels of IL17-A, as well. Finally, TH17 production was partially inhibited in the presence of anti-IL6 mAbs, or CTLA4-Ig fusion molecules, demonstrating thus the roles of IL6 and cell-cell interaction in such a production.

Conclusion We show herein, that interaction of MSC, or FLS from RA or OA patients, with MNC, or synovial T cells, results in enhancing the inflammatory response through production of IL-17-A, IL6, IL-8 and IL-1 $\beta$ cytokines. Moreover, in the context of an inflammatory environment, that is, the presence of IL-17-A, TNF $\alpha$, and/or IFN $\gamma$, such an interaction of MNC with MSC or FLS results in further amplifying the production of pro-inflammatory cytokines, leading thus to a vicious circle, that may participate in the chronicity of inflammation, in RA. 